\title{
YOUNG STAR CLUSTERS IN THE LMC
}

\author{
K.C. FREEMAN \\ Mt. Stromlo and Siding Spring Observatories \\ The Australian National University, ACT, Australia \\ R.A.W. ELSON \\ The Bunting Institute \\ Radcliffe College, Cambridge Ma. 02138, USA
}

\begin{abstract}
We discuss the integrated colours, kinematics, formation, dynamical evolution and initial mass functions of the young globular star clusters in the Large Magellanic Cloud (LMC). Because these clusters are so young, they offer us insights, unobtainable from the old globular clusters in our Galaxy, into the formation and early dynamical evolution of globular clusters.
\end{abstract}

\section{Introduction}

These young clusters have several designations: young populous clusters, blue globular clusters and young globular clusters. We will concentrate here on the brightest examples, such as NGC $1831,1866,2164,2214$ and the 30-Dor cluster. In mass and binding energy, they are like average globular clusters in the Galaxy: their masses are typically $\$ 10^{5} \mathbf{M}_{0}$ and their binding energies per unit mass (are a few $\left.\mathrm{km} \mathrm{s}^{-1}\right)^{2}$. However, with ages of about $5 \times 10^{6}$ to $3 \times 10^{8}$ years, these blue clusters in the LMC are much younger than the galactic globular clusters, and they have no counterparts in the Galaxy. Similar young populous clusters are found in the late type spiral M33 (Christian \& Schommer, 1982).

\section{Early history}

Shapley and Nail (1951) discovered cepheids in NGC 1866 (one of the brightest of the young globular clusters), and recognized that some of the globular-like clusters in the LMC were different from the halo globular clusters of our Galaxy. Gascoigne and Kron (1952) measured integrated PV colours for a sample of Magellanic Cloud clusters, and found two groups, one with colours normal for galactic globular clusters, and the other a blue group with $\mathrm{P}-\mathrm{V}<0.25$. More recent work on these objects began with Hodge's (1961) catalogue of 23 young populous clusters.

\section{Integrated colours}

In the (U-B) - (B-V) plane, the young clusters follow a well-defined locus which represents the ageing of their stellar content: the cluster age is tightly related to its position along this locus (see, for example, Elson \& Fall, 1985). The distribution of the bright clusters in the UBV plane is 
affected by their birthrate history, fading of their integrated light with age, and the disruption of the clusters by various dynamical processes. Together, these effects lead to a bimodal distribution of B-V for the bright clusters (e.g. van den Bergh \& Hagen, 1968). The implications of this bimodal colour distribution for the cluster formation rate are not yet fully understood (e.g. whether it implies bursts or lapses in the cluster formation history). It is clear that there are relatively more older clusters (ages $\gtrsim 10^{9}$ years) in the LMC than inthe open cluster population of the Galaxy (Elson \& Fall, 1985).

\section{Kinematics}

These young clusters are extreme Population I objects, and we would expect them to have kinematics similar to that of the HI in the LMC. This is indeed the case, as shown by Freeman et al. (1983), and confirmed by more recent work (see Schommer's review (1990)): the systemic velocity, kinematic line of nodes, and the amplitude of the rotational velocity field for the young cluster system in the LMC are all similar to those found for the HI.

\section{Formation}

The formation of young populous clusters (YPCs), like those in the LMC, has recently been discussed by Kennicutt and Chu (1988). Here are some points from their discussion, with some added comments.

- The 30-Dor cluster is an example of a YPC at an early stage of its life: the stellar mass in this YPC is $>$ several $\times 10^{4} \mathrm{M}_{\odot}$.

- The brightest giant HII regions in disk and irregular galaxies are ionized by very luminous stellar aggregates, with absolute magnitudes MB in the range -12.5 to -14 . For these giant HII regions, typically as bright or brighter than 30 Dor, the ionizing aggregate is frequently a compact YPC. The less luminous HII regions, like those in the Galaxy, are ionized by loosely bound associations. This indicates that the formation of giant HII regions and YPCs are related.

- Giant HII regions more luminous than 30 Dor are much more frequent in late-type spiral and irregular galaxies. No such HII regions are known in the Galaxy.

- Some dwarf irregular galaxies, like NGC 1705 (see Meurer et al. 1989), have single very luminous $\left(M_{B} \approx-14\right)$ compact YPCs, with masses of about $10^{6} \mathrm{M}_{\odot}$. There may be a link here between the formation of YPCs now in late-type galaxies like the LMC and the formation long ago of the galactic halo globular clusters. Searle and Zinn (1978) have argued that the halo clusters formed in gas-rich dwarf galaxies which were then accreted by the Galaxy NGC 1705 would appear to be a suitable prototype for the Searle-Zinn gas-rich dwarfs. The same processes that are acting now to form YPCs in the LMC and NGC 1705 may well have acted long ago in the SearleZinn gas-rich dwarfs to produce YPCs which we now recognize as galactic halo clusters.

\section{Dynamical evolution of young clusters}

Much has been done on the late stages of dynamical evolution of globular clusters, including the core collapse and post-collapse evolution: see Elson et al. (1987b) for a recent review. The early stages of dynamical evolution are also interesting, but are of course unobservable in the galactic globular clusters, which are all old. 
Chernoff and Weinberg (1990) investigated the dynamical evolution of globular clusters with a power law IMF ( $d N \alpha M^{-(1+x)} d M$ : the Salpeter function corresponds to $x=1.35$ ), including the following processes:

- collisional relaxation

- $\quad$ stellar evolution and the resulting mass loss

- the galactic tidal field. They showed that weakly concentrated clusters with flat IMFs disrupt within less than $10^{8}$ years through the combined effects of mass loss and the galactic tidal field. This mass loss can be very significant; for example, a cluster with $x=0.5$ loses about $60 \%$ of its mass in $3 \times 10^{7}$ years.

The young LMC clustes have ages of order $10^{7}$ to $10^{8}$ years, central relaxation times $\gtrsim 5=$ their ages (see Elson et al. 1987a) and orbital periods within the LMC of about $2 \times 10^{8}$ years. It follows that

- their dynamical evolution is dominated by mass loss, rather than by collisional relaxation, and

- we would expect that they are too young to be tidally truncated like the old galactic globular clusters. (Tidal truncation takes many orbital periods: see for example Seitzer 1985.)

\subsection{STRUCTURE OF YOUNG CLUSTERS}

This discussion follows Elson et al. (1987a); they measured the radial light distributions for several young globular clusters in the LMC, using photoelectric aperture photometry in the inner parts of the clusters and deep star counts from photographic plates in the outer parts. The wide field of the photographic plates ( 25 arcmin) allows accurate determination of the stellar background density around each cluster.

The surface brightness profiles for their clusters showed an inner core and an outer power law distribution, and were well represented by the simple function

$$
\mu(r)=\mu_{0}\left(1+r^{2} / a^{2}\right)^{-\gamma / 2}
$$

where $a$ is the core radius. For ten young clusters, the mean value of $\gamma$ is $2.7 \pm 0.4(\sigma)$. Then the volume density $\rho(r) \sim r^{3.7}$ in the outer regions: this is like the density law for elliptical galaxies, and is consistent with violent relaxation.

The surface brightness profiles for these young clusters show no evidence for a tidal cutoff: the clusters extend well beyond their eventual tidal radii (as estimated from their mass and the tidal field of the LMC. This result has recently been confirmed for NGC 1866 by Fischer et al. (1990).

\subsection{CORE EXPANSION IN YOUNG CLUSTERS}

Elson et al. (1989b) studied the cores of $18 \mathrm{LMC}$ globular clusters with ages in the range $10^{7}$ to $\gtrsim$ $10^{9}$ years, using short exposure B and V CCD images. They estimated the core radii for these clusters, using the fitting function given above, and combined them with estimates of core radii for another 17 LMC clusters from the literature to see how the core radii of these clusters change with age. Figure 1 shows the main results:

- all of the youngest clusters in their sample have small cores

- the mean core radius increases with age

- a few of the oldest clusters (ages $\sim 10^{10}$ years) have small cores, which suggests that they are approaching core collapse.

Comparison with the Chernoff-Weinberg (1990) models suggests that the IMF slope is about 1 for some of the clusters and is perhaps closer to 2 for some others. It seems unlikely that the older 
clusters in the sample have lower values of $x$, because clusters with $x \$ 0.5$ disrupt within about $3 \times 10^{7}$ years.

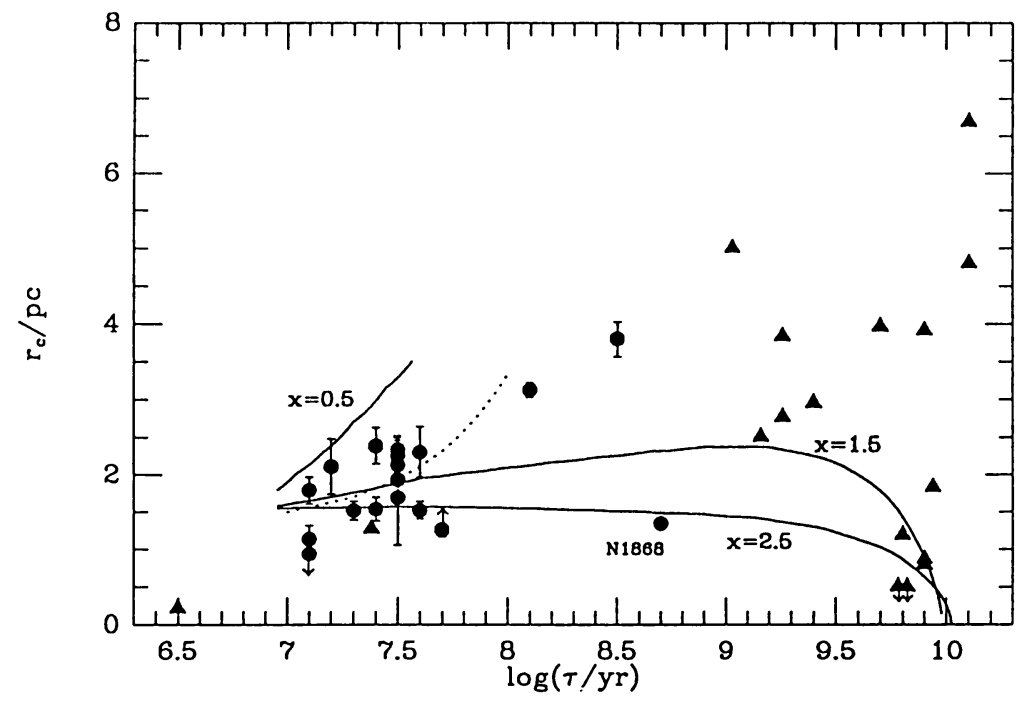

Figure 1. Core radius $v$. age for the clusters of Elson et al. (1989b). The solid curves are from Chernoff-Weinberg models of clusters, with $M=6 \times 10^{4} \mathrm{M}_{\odot}$, central potentials Wo. $=7$ (King, 1966), at 3 $\mathrm{kpc}$ from the centre of the LMC; the slopes of the power-law IMFs are indicated. The dotted curve represents constant expansion at $0.02 \mathrm{~km} \mathrm{~s}^{-1}$.

\section{Direct measurements of the IMF}

The IMF slope is an important parameter for understanding the chemical and dynamical evolution of these clusters, and also for understanding the impact of these clusters on their environment. The upper part of the IMFs for the young clusters can, in principle, be derived from their observed main sequence luminosity functions. The luminosity functions have been measured by several groups, using photographic and CCD methods, but there is significant disagreement. Each method has its own technical problems. The photographic approach has the advantage of large field and accurate background subtraction, and the disadvantage of nonlinearity and limited dynamic range. The CCDs used so far are small, and it has been necessary to work in the crowded inner regions of the clusters to get adequate statistics for the luminosity function; this in turn requires large and uncertain incompleteness corrections. Here is a summary of some recent results:

- Mateo (1988: CCD). Five LMC clusters and one SMC cluster with ages in the range $10^{7}$ to $2 \times 10^{9}$ years: $1.5 \leqslant x \leqslant 3.5$, but consistent with constant $x$.

- Elson et al. (1989a: photographic). Six LMC clusters with ages between $3 \times 10^{7}$ and $5 \times 10^{7}$ years: $-0.2 \$ x \leqslant 0.8$, again consistent with constant $x$.

- Richtler and de Boer (1989: CCD). Two LMC clusters with ages of $3 \times 10^{7}$ years: $x=2.5$ and 3.5.

- Richtler et al. (1990: CCD). Four young LMC clusters, $x$ in the range 0.5 to 1.5 .

At this stage, the results on the IMF slopes cannot be regarded as definitive. 


\section{Problems}

The slope of the IMF for the young clusters is an important but difficult observational problem. Wide-field CCD images will help, by giving accurate background correction and adequate statistics for the luminosity function, while avoiding the need to work in the crowded inner regions of the clusters.

Dynamical masses for the young clusters would be very useful for constraining the lower end of the IMF, and also for estimating how much the clusters overfill their eventual tidal limits. The measurements are difficult, because the internal velocity dispersions are small (typically $\leqslant 3 \mathrm{~km} \mathrm{~s}^{-1}$ ). On the other hand, analysis is more straightforward than for galactic globular clusters, because the relaxation times are longer than the cluster ages and so mass segregation effects should be small. For some recent progress on this problem, see Lupton et al. (1989) and the paper by Fischer et al. (1990) at this conference.

Several of the young globular clusters in the LMC have ages $\$ 10^{7}$ years, and it seems likely that there would be several protoclusters in the phase immediately preceding star formation. It would be interesting to find ways to detect such protoclusters.

\section{References}

Chernoff, D., Weinberg, M. (1990), Astrophys. J. 351, 121.

Christian, C.A., Schommer, R.A. (1982), Astrophys. J. Suppl. 49, 405.

Elson, R.A.W., Fall, S.M. (1985), Astrophys. J. 299, 211.

Elson, R.A.W., Fall, S.M., Freeman, K. (1987a), Astrophys. J. 323, 54.

Elson, R.A.W., Hut, P., Inagaki, S. (1987b), Ann. Rev. Astron. Astrophys. 25, 565.

Elson, R.A.W., Fall, S.M., Freeman, K.C. (1989a), Astrophys. J. 336, 734.

Elson, R.A.W., Freeman, K., Lauer, T.R. (1989b), Astrophys. J. 347, L69.

Fischer, P., Welch, D.L., Cuic, P., Mateo, M.L., Madore, B.F. (1990), these proceedings.

Freeman, K.C., Illingworth, G., Oemler, A. (1983), Astrophys. J. 272, 488.

Gascoigne, S.C.B., Kron, G.E. (1952), Publ. Astron. Soc. Pac. 64, 196.

Hodge, P. (1961), Astrophys. J. 133, 413.

Kennicutt, R.C., Chu, Y.-H. (1988), Astron. J. 95, 720.

King, I.R. (1966), Astron. J. 71, 64.

Lupton, R.H., Fall, S.M., Freeman, K.C., Elson, R.A.W. (1989), Astrophys. J. 347, 201.

Mateo, M. (1988), Astrophys. J. 331, 261.

Meurer, G.R., Freeman, K.C., Elson, R.A.W. (1989), Astrophys. J. 347, 201.

Mateo, M. (1988), Astrophys. J. 331, 261.

Meurer, G.R., Freeman, K.C., Dopita, M.A. (1989), Astrophys. Space. Sci. 156, 141.

Richtler, T., de Boer, K.S. (1989), Recent Developments of Magellanic Cloud Research, K.S. de Boer et al. (eds.) (Observatoire de Paris: Paris) p.91.

Schommer, R.A., Olszewski, V.E.W., Suntzeff, N.B., Harris, H.C. (1990), these proceedings. Searle, L., Zinn, R. (1978), Astrophys. J. 225, 357.

Seitzer, P. (1985), IAU Symp. 113 Dynamics of Star Clusters, J. Goodman \& P. Hut (eds.)

(Reidel: Dordrecht) p.343.

Shapley, H., Nail, V. (1951), Astron. J. 55, 249.

van den Bergh, S., Hagen, G.L. (1968), Astron. J. 73, 569. 